\title{
Die Kartografie radikaler Demokratietheorien
}

\author{
Milos Rodatos ii
}

Angenommen: 7. Januar 2021 / Online publiziert: 27. Januar 2021

(C) Der/die Autor(en) 2021

Comtesse, Dagmar, Oliver Flügel-Martinsen, Franziska Martinsen, und Martin Nonhoff (Hrsg.) (2019): Radikale Demokratietheorie. Ein Handbuch. Berlin: Suhrkamp. 832 Seiten. $30,00 €$

Flügel-Martinsen, Oliver (2020): Radikale Demokratietheorien zur Einführung. Hamburg: Junius. 188 Seiten. 14,90€

Mit den hier rezensierten Erscheinungen hat die Etablierung eines eigenständigen radikaldemokratischen Paradigmas innerhalb der Demokratietheorie einen eindrucksvollen Meilenstein erreicht. Beide Werke forcieren, trotz argumentativer und personeller Verschränkung (Flügel-Martinsen, S. 12 Fn. 1), zwei sehr unterschiedliche Strategien zur Kartografie radikaldemokratischer Theorien, deren vielen Stärken und wenigen Unzulänglichkeiten ich im weiteren Verlauf diskutieren werde. Während das Handbuch die Rekonstruktion der Vielfalt und Heterogenität radikaler Demokratietheorien in den Vordergrund stellt, präsentiert Oliver Flügel-Martinsen in seiner Einführung eine sehr bestimmte Lesart radikaldemokratischer Ansätze. Ich werde dies an seiner Diskussion des Kontingenzbegriffs und dem Verhältnis von radikaler Demokratie und Populismus verdeutlichen.

Zunächst aber zum Aufbau des Handbuchs: Dieses umfasst insgesamt 83 Einträge, die sich in vier Teilabschnitte gliedern. Im ersten, ideengeschichtlich orientierten Teil werden wichtige Einflüsse für das radikaldemokratische Denken nachverfolgt. Unter dem Schlagwort „Vorläufer*innen“ werden AutorInnen diskutiert, deren Werk „,entweder erste Denkanstöße und Ideen zu einer Theorie radikale Demokratie liefert oder [die] selbst bereits radikaldemokratisch relevante Theoreme vertreten" (Comtesse et al., S. 16). Das Handbuch beschränkt sich dabei nicht nur auf bereits bekannte

M. Rodatos $(\bowtie)$

Universität Greifswald, Greifswald, Deutschland

E-Mail: milos.rodatos@uni-greifswald.de 
ideengeschichtliche Pfade, sondern präsentiert beispielsweise mit John Dewey (Jörke und Selk, S. 78-87) sowie mit Beiträgen unter dem Label „Autor*innen jenseits des Kanons“ Anknüpfungspunkte, die bisher weniger Aufmerksamkeit im radikaldemokratischen Denken erfahren haben.

Im zweiten Teil werden gegenwärtige Positionen radikaler Demokratie vorgestellt, wobei die HerausgeberInnen hier drei Auswahlkriterien benennen: Erstens eine weitreichende Kontingenzannahme für das gesellschaftliche Zusammenleben und dessen jeweilige (institutionalisierte) Ausgestaltung; zweitens das Plädoyer für eine ausgeprägte kritische Selbstreflexion, in der das Infragestellen von Praktiken, Normen und Institutionen in den Vordergrund tritt; und drittens das Eintreten für die Intensivierung und Ausweitung demokratischer Selbstregierung (S. 12-15). Diese Kriterien werden von den HerausgeberInnen im Eintrag zum Begriff der Demokratie noch einmal ausführlicher beleuchtet, ohne dabei eine engere Definition des Demokratiebegriffs oder eine Typologie radikaldemokratischen Denkens zu forcieren (S. 457-483). Vielmehr geht es um die Schaffung von genuin radikaldemokratischen Orientierungspunkten im Demokratiediskurs, welcher ,,als begriffliches wie praktisches Konfliktfeld“ verstanden wird (S. 457). Innerhalb dieses Konfliktfeldes wird der Geltungsbereich radikaldemokratischen Denkens weit abgesteckt und die HerausgeberInnen beweisen eine ausgeprägte Sensibilität gegenüber der Vielfalt des Forschungsfeldes, indem TheoretikerInnen mitaufgenommen wurden, die sich dezidiert nicht als RadikaldemokratInnen verstehen oder aber von solchen als GegnerInnen verstanden werden. Die dadurch entstehenden Ambivalenzen werden in den Beiträgen transparent verhandelt, wie exemplarisch die Einträge zu Jürgen Habermas (Nonhoff, S. 294-303) oder Slavoj Žižek (Heil, S. 444-452) zeigen.

Die letzten beiden Teilabschnitte des Handbuchs diskutieren grundlegende Begriffe, Themenfelder sowie gegenwärtige Kontroversen innerhalb des radikaldemokratischen Terrains und bieten eine gute Kontrastfolie für die Diskussion von FlügelMartinsens Arbeit. Dessen Einführung soll ,zusammenhängend in Gemeinsamkeiten, in Konflikte unter den Positionen und in Abgrenzungen zu anderen Ansätzen einführen und so ein lebendiges Panorama des Diskurses radikaler Demokratietheorien entwerfen“ (S. 12). Flügel-Martinsen entscheidet sich für eine kompakte Einführung, indem er die konkrete Wegeführung in das radikaldemokratische Terrain anhand von Begriffsverständnissen, Themen- und Spannungsfeldern vollzieht. Dabei kommt es an manchen Punkten zur bereits erwähnten argumentativen Verschränkung zwischen Handbuch und Einführung, was beispielsweise bei den radikaldemokratischen Lesarten von Jürgen Habermas und Richard Rotry deutlich wird, wobei Flügel-Martinsen an dieser Stelle stärker deren Kontingenzbegriff ,[im Sinne] eines Verlusts von feststehenden Orientierungspunkten“ (S. 47) in gegenwärtigen Gesellschaften forciert (S. 49-58). Hierbei gelingt Flügel-Martinsen die prägnante und klare Differenzierung radikaldemokratischer Perspektiven auf diese Kontingenzdiagnose. Dessen starke Lesart verbindet er dabei mit dem Konzept der politischen Differenz von Oliver Marchart, die als ,eine allgemeine (politische) Theorie der Kontingenz" verstanden wird und ,die es ermöglicht, die Möglichkeit letzter Gründe generell in Zweifel zu ziehen“ (S. 66). Flügel-Martinsen begreift dies als eine „Quasi-Transzendentalie“ (S. 66), die in Konsequenz zu einer konfliktiven Verfasstheit des Politischen auf ontologischer Ebene führt (S. 116-118). Er steht einer solchen 
„politischen Ontologie des Konflikts“ (S. 114) skeptisch gegenüber und attestiert der starken Lesart Widersprüchlichkeit zu den eigenen postessenzialistischen Prämissen (S. 119). Erfolgversprechender erscheint ihm deshalb eine schwache Lesart der Kontingenzdiagnose, welche genealogisch und dekonstruktivistisch arbeitet, ohne dabei auf eine allgemeine Theorie der Kontingenz zu verweisen (S. 67-71). Der Konflikt ist so nicht auf ontologischer Ebene, sondern in der spezifischen politischen Praxis verortet (S. 121). Wenngleich Flügel-Martinsen betont, dass diese Praxis der Infragestellung keine „Automatismen“ impliziert, kommt er zu dem Ergebnis, dass „Dekonstruktion und radikale Demokratie [...] Praktiken der Infragestellung [sind], die sich von noch so stabil abgesicherten Ordnungen nicht gänzlich ausschließen lassen“ (S. 122). Hier bleibt der tatsächliche Unterschied allerdings unklar, denn beide Kontingenzverständnisse verweisen gleichermaßen auf die politikpraktische Unmöglichkeit einer bis ins letzte Glied stabilisierten Ordnung.

Es gelingt Flügel-Martinsen nichtsdestotrotz durch seine affirmative und offen dargelegte Positionierung für die schwache Lesart der Kontingenzdiagnose, Demarkations- und Spannungslinien zwischen radikaldemokratischen Ansätzen kompakt darzustellen. Die Verknüpfung von Kontingenzbegriff und Konfliktverständnis lässt sich im Handbuch dagegen in dieser Tiefe nur indirekt nachverfolgen (u.a. im Handbuch Oppelt und Sörensen, S. 139-143; Vasilache, S. 492-503; Marchart, S. 572-575; und Flügel-Martinsen, S. 578-581), was keine Unzulänglichkeit bedeutet, sondern dem Charakter eines Handbuchs geschuldet ist.

Mit Blick auf Flügel-Martinsens Einordnung des Verhältnisses zwischen radikaler Demokratie und Populismus besteht jedoch die Gefahr, produktive Spannungspotenziale zu verspielen. Er begreift zwar leichtfertige Populismusvorwürfe als ,(neo-)liberale Delegitimierungsstrategie“ (S. 143-144), die auch zur Diskreditierung radikaldemokratischer Praktiken der Infragestellung führen kann. Es folgt dann aber eine rigorose Immunisierungsstrategie für das radikaldemokratische Denken gegenüber populistischen Praktiken, die bei der Konstruktion eines Kollektivakteurs im nationalstaatlichen Register dem ,Salonfähigwerden nationalistischer Argumente Vorschub [...] leisten“ (S. 154) würden. Flügel-Martinsen sieht keine gewinnbringende Unterscheidung zwischen Rechts- und Linkspopulismus, weil beide die Konstitution eines Kollektivakteuers mit Akten der Exklusion verknüpfen ,und eben nicht als Bezugspunkt einer pluralistischen Konstitution kollektiver Identität geeignet [sind]“ (S. 153). Radikale Demokratie, verstanden als Verbindung aus einer ,,sozio-ökonomischen linken Forderung nach Umverteilung“ und ,kultur- und ökolinken Argumenten“ (S. 160-161), wird in „,einer inklusiven und nicht in einer exklusiven Logik“ (S. 162) praktiziert und ist deshalb für Flügel-Martinsen nicht vereinbar mit populistischen Praktiken, die sich affirmativ auf nationalstaatliche Zugehörigkeiten beziehen und dadurch exkludierend wirken (S. 159-161).

Diese Einordnung zwingt ihn dazu, Ernesto Laclau und Chantal Mouffe, die beide auf die Notwendigkeit der Exklusion in der hegemonialen Konstruktion eines Kollektivakteurs abstellen, denen er aber weiterhin eine wichtige Rolle für das radikaldemokratische Denken zugesteht (S. 145, 148), eine politiktheoretische Doppelrolle zuzuweisen. Für Laclau gilt es, zwischen ,dem radikaldemokratischen Theorierahmen im engeren und dem hegemonietheoretischen Ansatz im weiteren Sinne zu unterscheiden“ (S. 145). Hier lässt sich mit dem Handbucheintrag zu Laclau 
einwenden, dass Laclau selbst sein hegemonietheoretisches Populismusverständnis im spannungsreichen, aber eben auch produktiven Verhältnis zu seinen radikaldemokratischen Überlegungen verortet (Nonhoff, S. 344-345). Mouffes Doppelrolle rekonstruiert Flügel-Martinsen, indem er ihre radikaldemokratischen Prämissen von ihrer affirmativen Zuwendung zum Linkspopulismus abgrenzt, die Mouffe nicht als radikaldemokratische Theoretikerin, sondern als ,theoretisch-praktische Akteurin“ vollzieht (S. 150). Aus meiner Sicht droht mit Flügel-Martinsens Bestimmung radikaldemokratischen Denkens auf der Grundlage einer sehr konkreten Vorstellung davon, was ein solches Denken als politisch links auszeichnet, eine zu einseitige Verortung des Verhältnisses von radikaler Demokratie und Populismus. Aristotelis Agridopoulos und Seongcheol Kim zeichnen im Handbucheintrag zu Populismus demgegenüber ein differenziertes Bild. So verdeutlichen beide, dass im Anschluss an Laclaus Populismustheorie bereits die Möglichkeiten einer emanzipatorischen und demokratischen Konstitution ausgelotet werden und somit die Bedingungen, „wie das Volk konstruiert wird“ (S. 596), in den Fokus rücken.

Flügel-Martinsens Immunisierungsstrategie wirft damit die Frage auf, wie gewinnbringend der Versuch ist, bestimmte Positionen aus dem radikaldemokratischen Terrain zu exkludieren, weil ihnen problematische Konsequenzen in der politischen Praxis zugeschrieben werden. Vielversprechender erscheint es mir, seine vorgebrachte Kritik noch stärker auf Ebene der realpolitischen Umsetzung radikaler Demokratie zu verorten und weniger im Register der Zugehörigkeit zum radikaldemokratischen Terrain. Hier ist der Handbuchbeitrag zum Verhältnis von Theorie und Praxis hilfreich, der auf die Notwendigkeit einer ,praxeologische[n] Wendung“ radikaler Demokratietheorie verweist (Süß, S. 794). Flügel-Martinsens Rekonstruktion einer antiinstitutionellen, einer im Anschluss an Jacques Rancière an-archischen und einer strategisch-affirmativen Perspektive radikaler DemokratInnen auf demokratische Institutionen kann dann als Ausgangspunkt fungieren (S. 123-128), um zu überprüfen, welche Ambivalenzen und Spannungen zwischen Theorien und Praktiken radikaler Demokratie bestehen. Dies erscheint mir zielführender, weil wir so die gegenwärtige Debatte zum Verhältnis von radikaler Demokratie und (demokratischen) Institutionen strukturieren und damit bearbeitbarer gestalten.

Wenngleich ich es mit Blick auf die Spannungslinien und Heterogenität des radikaldemokratischen Terrains für produktiv halte, die sehr bestimmte Lesart der Einführung mit verschiedenen Handbuchbeiträgen zu kontrastieren, möchte ich abschließend betonen, dass sowohl das Handbuch als auch die Einführung wesentliche Bereicherungen für die Forschung und Lehre mit radikaldemokratischen Ansätzen bieten und deshalb unbedingt Einzug in unsere Curricula und Bibliotheken finden sollten. Den beteiligten AutorInnen gilt es zu danken für fundierte Orientierungspunkte im radikaldemokratischen Terrain, die für Debatten mit und gegen radikale Demokratietheorien wichtige Impulsgeber sein werden.

Funding Open Access funding enabled and organized by Projekt DEAL.

Open Access Dieser Artikel wird unter der Creative Commons Namensnennung 4.0 International Lizenz veröffentlicht, welche die Nutzung, Vervielfältigung, Bearbeitung, Verbreitung und Wiedergabe in jeglichem Medium und Format erlaubt, sofern Sie den/die ursprünglichen Autor(en) und die Quelle ord- 
nungsgemäß nennen, einen Link zur Creative Commons Lizenz beifügen und angeben, ob Änderungen vorgenommen wurden.

Die in diesem Artikel enthaltenen Bilder und sonstiges Drittmaterial unterliegen ebenfalls der genannten Creative Commons Lizenz, sofern sich aus der Abbildungslegende nichts anderes ergibt. Sofern das betreffende Material nicht unter der genannten Creative Commons Lizenz steht und die betreffende Handlung nicht nach gesetzlichen Vorschriften erlaubt ist, ist für die oben aufgeführten Weiterverwendungen des Materials die Einwilligung des jeweiligen Rechteinhabers einzuholen.

Weitere Details zur Lizenz entnehmen Sie bitte der Lizenzinformation auf http://creativecommons.org/ licenses/by/4.0/deed.de. 